\title{
Travel Time Estimation Model Based on Markov Queuing Model
}

\author{
Zhijian Wang ${ }^{1, a}$, Zhengying Hou ${ }^{1, b}$ and Haoran Liu $^{2, c}$ \\ ${ }^{1}$ Intelligent Transportation Institute, North China University of Technology, Beijing 100041, China \\ ${ }^{2}$ School of Mathematical Science, Peking University, Beijing 100871, China \\ aemail:wzjian0722@163.com, bemail:houzhengyingzdh@163.com, \\ cemail:pku2014liuhaoran@163.com
}

Keywords: Markov chain; travel time estimation; traffic wave theory; stochastic arrivals

Abstract. To overcome the limitation of the existing models intersection queuing models in which vehicle arrivals are assumed to meet some specific traffic distribution, a new model which based on markov chain and traffic wave theory is proposed to estimate the travel time of vehicles. This model fits the signalized intersection with fixed signal cycle and stochastic vehicle arrivals. At last, the model has experimented on a road of Beijing and the results shows that it has high estimation precision.

\section{Introduction}

Link travel time is an important index to reflect the traffic condition. Therefore, accurate and real-time road travel time is an crucial content of traffic guidance and signal control, which is of great significance. Link travel time is the most direct parameter to characterize the amount of resistance encountered by a vehicle during its travel, So it is also a link impedance. So the link travel time estimation model is also known as the link resistance function.

There are three types of travel time estimation methods: One is based on mathematical statistics, including linear regression[1] and nonlinear regression[2], Kalman filtering[3] and Bayesian estimation [4]; One is based on intelligent theory [5-6]; There is also a class of simulation analysis model [8-10].

These models consider less interrupted traffic and the impact of the signal timing scheme, require more parameter calibration, data types, data volume, and the traffic flow to meet a certain distribution. These cannot be a good response to urban road realities of interrupted traffic. In this paper, the travel time estimation model is established by the Markov queuing model [11] based on the stochastic arrival of vehicles and the traffic wave theory, considering the intersection of the queuing situation and the intersection delay.

\section{Link travel Time Estimation Model}

Travel time estimation of link area

In general, the link of road refers to the extension line from the extension line of the stop line of the last intersection to the exit line of the next intersection, which can be divided into intersection area and link area.

Because the travel time of the link area is relatively stable, it is a continuous flow and the GPS data of the stable section can be used to get the better results.

Travel Time Estimation Model of Intersection Area

While the intersection area involves traffic lights-red light, the vehicle may be queued at the intersection, thus forming an interrupted flow. On the one hand the intersection is similar to the multiple single-channel service model, and on the other hand it has traffic wave characteristic by the role of intermittent flow.

This paper established the model of intersection interrupted flow based on the Markov queuing theory and traffic flow wave theory, which was combining with the signal timing scheme.

Link travel time consists of travel time of link area and that of intersection area.The travel time of intersection area can be divided into the following two parts according to the distance of the vehicle away from the intersection and its arrival time. 
Case (1) :vehicles in red and yellow light time reach the intersection, decelerate until the stop queue, and then accelerate through the stop line in the green light time.

Case (2): vehicles in green time reach the intersection, decelerate to queue but unstop, and then accelerate through the stop line.

For the case (1), the queuing model of signalized intersection is established based on the improved Markov chain method, and estimates the queuing time of the vehicle according to the signal timing scheme of the intersection.

For the (2) case, we can estimate travel time by the collection of vehicle GPS speed, location and other information.

Data Acquisition of Markov Chain Queue Model

The following data are collected from import road:

(1) Green time $t_{g}$, Yellow light time $t_{y}$, Red light time $t_{r}$, Yellow and red light time $t_{a}$, $t_{a}=t_{y}+t_{r}$.

(2) Loss of time $t_{l}$.

(3) The number of lanes of the import lane $n$, the $x$ lane saturation headway $h_{x}$.

(4) Import Road Capacity $Q$, which allows the largest number of vehicles queuing

(5) The number of vehicles arriving at the green light time of the $m$ cycle of the investigation phase $a_{1}$, the number of vehicles arriving at yellow, red light time $a_{2}$, the total number of cycles measured $M$.

Calculate the following values:

(1) The maximum number of vehicles crossing the intersection during the effective green time $s$ can be obtained in Eq.1. $s$ also is the maximum traffic flow what is the maximum flow rate in the effective green time through the intersection。

$$
s=\left(t_{g}+t_{y}-t_{l}\right) / \sum_{x=1}^{n} h_{x} .
$$

(2) the probability of $\mathrm{k}$ vehicles reach the intersection in green light time $p_{g a k}$, the probability of $\mathrm{k}$ vehicles reach the intersection in yellow, red light time $p_{\text {yrak }}$. These can be learned through statistical data。

Markov chain queuing model

Markov process is characterized by the transfer of each state are only connected with the previous state of a state, regardless of the state of the past, what also is no after-effect. The traffic flow fits the Markov chain very well. The queue length is the longest at the critical moment when the red light ends, so we only need to analyze the queuing law at this momento

Step 1 Determine the state transition matrix $p_{i j}$ :

Assuming that the number of vehicles queued at the end of the current red light is $i$, then the probability of transferring to the queued state $j$ after one cycle is given by Eq.2.

$$
\begin{aligned}
& p_{i j}=P\left(\max \left(i+V_{g a}-s, 0\right)+V_{y r a}=j\right), \\
& p_{i Q}=P\left(\max \left(i+V_{g a}-s, 0\right)+V_{y r a} \geq Q\right), \\
& 0 \leq i \leq Q, \\
& 0 \leq j<Q, \\
& p_{i j} \geq 0, \\
& p_{i Q} \geq 0,
\end{aligned}
$$


In above equation, $p_{i j}$ is the probability that the number of vehicles queuing up is shifted from $i$ to $j$ by one step,$V_{g a}$ is the random variable of vehicles arriving at the entrance lane during the green time, $V_{y r a}$ is the random variable of vehicles arriving at the entrance lane in yellow, red light time。

The above formula shows that if $i+V_{g a}-s>0$, then the number $j$ of next red light at the end of the queue is equal to $i+V_{g a}-s+V_{y r a}$. That is, if the current queue number $i$ of red light vehicles plus the number $V_{g a}$ of vehicles to reach the intersection in the green light more than the maximum number $s$ of vehicles to cross the intersection in the effective green time, then $j$ equals $i+V_{g a}-s+V_{y r a}$.

In other words, if the import lane in the green light time cannot be empty, $j$ is equal to $i$ plus $V_{g a}$ plus $V_{y r a}$ minus $s$; Otherwise, if the import lane in the green light time can be empty, then $j$ equals $V_{y r a}$. Subsequent vehicles will not be queued in this intersection area if $j=Q$. So Eq.3 can be obtained by further development.

$$
\begin{aligned}
& p_{i j}=\sum_{k=0}^{s-i} p_{g a k} p_{y r a k}+\sum_{k=s-i+1}^{s} p_{g a k} p_{y r a(j+s-i-k)}, \\
& p_{i Q}=\sum_{k=0}^{s-i} p_{g a k} p_{y r a(\geq Q)}+\sum_{k=s-i+1}^{s} p_{g a k} p_{y r a(\geq Q+s-i-k)}, \\
& 0 \leq i \leq Q, \\
& 0 \leq j<Q, \\
& p_{\text {gak }}=0, k<0 \text { or } k>\max _{1 \leq b \leq M}\left\{V_{g a b}\right\}, \\
& p_{y r a k}=0, k<0 \text { or } k>\max _{1 \leq b \leq M}\left\{V_{y r a b}\right\},
\end{aligned}
$$

if $i>s$, then Eq.4 can be concluded.

$$
\left.\begin{array}{l}
\sum_{k=0}^{s-i} p_{g a k} p_{y r a(\geq Q)}=0, \\
\sum_{k=s-i+1}^{s} p_{g a k} p_{y r a(\geq Q+s-i-k)}=\sum_{k=0}^{s} p_{g a k} p_{y r a(\geq Q+s-i-k)}, \\
p_{y r a(\geq Q)}=\sum_{k=Q}^{\infty} p_{y r a k}, \\
p_{y r a(\geq Q+s-i-k)}=\sum_{b=Q+s-i-k}^{\infty} p_{y r a b}
\end{array}\right\}
$$

The matrix of queuing state transition probability can be obtained from the statistic data of the intersection.

Step 2 Determine the steady-state probability:

From the queuing model of the principle of equal speed, The probability that the intersection changes from the state of the number $j$ of queuing vehicles at the end of the previous red light to the state of the number $i$ of queuing vehicles at the end of the current red light is equal to the probability of that from $i$ to the state of the number $j$ of vehicles queued at the end of the next red light, which is shown in Eq.5.

$$
\sum_{j \neq i} p_{i} p_{i j}=\sum_{i \neq j} p_{j} p_{j i}, 0 \leq i<Q, 0 \leq j \leq Q
$$

The balanced equation can be concluded in Eq.6 and Eq.7. 


$$
\begin{aligned}
& p_{i}=\sum_{j} p_{j} p_{j i}, 0 \leq i<Q, 0 \leq j \leq Q \\
& \sum_{i} p_{i}=\sum_{j} p_{j}=1
\end{aligned}
$$

Eq. 8 can be obtained to sum up Eq.2 Eq.7.

$$
\begin{aligned}
& p_{i j}=\sum_{k=0}^{s-i} p_{g a k} p_{y r a k}+\sum_{k=s-i+1}^{s} p_{g a k} p_{y r a(j+s-i-k)}, \\
& p_{i Q}=\sum_{k=0}^{s-i} p_{g a k} p_{y r a(\geq Q)}+\sum_{k=s-i+1}^{s} p_{g a k} p_{y r a(\geq Q+s-i-k)}, \\
& 0 \leq i \leq Q, \\
& 0 \leq j<Q, \\
& p_{g a k}=0, k<0 \text { or } k>\max _{1 \leq b \leq M}\left\{V_{g a b}\right\}, \\
& p_{y r a k}=0, k<0 \text { or } k>\max _{1 \leq b \leq M}\left\{V_{y r a b}\right\}, \\
& \sum_{i} p_{i}=1
\end{aligned}
$$

$p_{i}$ can be obtained in Eq.9.

$$
p_{i}=\frac{D(i+1)}{D}, i=0,1, \mathrm{~L}, Q
$$

In the Eq.9, $D$ can be obtained from the following Eq.10.

$$
D=\left|\begin{array}{ccccc}
1 & 1 & \mathrm{~L} & 1 & 1 \\
p_{00}-1 & p_{10} & \mathrm{~L} & p_{Q-1,0} & p_{Q 0} \\
\mathrm{M} & \mathrm{M} & \mathrm{M} & \mathrm{M} & \mathrm{M} \\
p_{0, Q-1} & p_{1, Q-1} & \mathrm{~L} & p_{Q-1, Q-1}-1 & p_{Q-1, Q-1}
\end{array}\right|
$$

The average queue length can be obtained from the following Eq.11 wherein $\bar{A}$ is the average vehicle length.

$$
\bar{L}=\bar{A} \sum_{i=1}^{Q} i p_{i}
$$

Traffic flow wave model

The basic equation of traffic flow wave model is Eq.12.

$$
V_{w}=\frac{q_{2}-q_{1}}{k_{2}-k_{1}}
$$

In the Eq.12, 1,2 refers to two different densities on the road, wherein a, b refers to the first traffic flow and density and c, d refers to the second traffic flow and density.

For case (1), the first traffic flow is 0 , and the density is $k$, so Eq.13 can be obtained by the Green Shields Speed-Density Linearity Model.

$$
v_{i}=v_{f}\left(1-\frac{k_{i}}{k_{j}}\right)
$$


So for the starting wave in the Theory of Traffic, $v_{1}=0, k_{1}=k_{j}$ when the cars are in the queue. In the green traffic lights, so we can get Eq.14 by taking $v_{1}, k_{j}, v_{2}$ to Eq.12 and Eq.13.

$$
V_{w}=-\left(v_{f}-v_{2}\right)
$$

In the Eq.14, $v_{2}$ is always very low and negligible.

Time headway will be relatively stable in the discharge of the front 8 to 10 queuing vehicles[12], because different speed stratifications occur when queuing vehicles passed stop line. The first layer of vehicles can achieve the speed that is close to or greater than the design speed because of no vehicles in front of this layer.

So $v_{f}$ is appropriate to take the speed of the second wave vehicle through the stop line, and it can be obtained by the GPS statistics data of floating car data.

By vehicle queue length $\bar{L}$, vehicle speed of the starting wave $V_{w}$, the travel time of the vehicle in the green time through the intersection can get from Eq.15.

$$
t_{1}=\frac{\bar{L}+L_{1}}{V_{w}}
$$

In the Eq.15, $L_{1}$ is the distance between the stop line of entrance lane and the extension line of the exit lane stop line。

Because the intersection signal timing is fixed, there are $\beta$ cycle the day. $\beta$ can be obtained by Eq.16 wherein $C$ is traffic signal cycle.

$$
\beta=\frac{24 \times 3600}{C}
$$

When the vehicle enters the queue at the red light time, the vehicle queuing time $t^{\prime}$ shown in Eq.17 can be derived from the time difference between the vehicle arrival time $t_{\text {arr }}$ and the next green light to the current lane signal cycle.

$$
t^{\prime}=\alpha C-t_{g}-t_{\text {arr }}, \alpha=0,1,2, \mathrm{~L}, \beta
$$

In the above formula, $t_{\text {arr }}$ shown in Eq.18 is the time that the vehicle arrived at the intersection. In Eq.18, $P_{v e h}$ is the current GPS position of the vehicle and $P_{i n s e c}$ is the GPS position of the next intersection.

$$
t_{\text {arr }}=\frac{P_{v e h}-P_{i n s e c}}{\bar{V}}
$$

Therefore, the travel time of the vehicle through the intersection area is shown in Eq.19.

$$
t_{1 \text { all }}=t_{1}+t^{\prime}
$$

The travel time of the vehicle through the link area is shown in Eq.20, wherein $L_{\text {road }}$ is the length of the link and $\bar{V}$ is the average running speed that can be get by the GPS statics data.

$$
t_{2}=\frac{L_{\text {road }}}{\bar{V}}
$$

For case (2), the travel time can be obtained by the information like the speed and position of the vehicle, wherein $L_{2}$ is the distance away from the stop line in the case of (2). 
$t_{3}=\frac{L_{1}+L_{2}}{V_{w}}$

So the total travel time estimation model of the vehicle through the link is:

$t_{\text {all }}=\left\{\begin{array}{l}t_{1 \text { all }}+t_{2}, \text { the vihicles arrived at the intersection in yellow, red light time } \\ t_{3}+t_{2}, \quad \text { the vihicles arrived at the intersection in green time }\end{array}\right.$

\section{Experiment}

In this paper, Fushi Road between East Bajiao Street and East Yangzhuang Street is taken as the research object showed in Fig.1. The road area is from the intersection of East Bajiao Street and Fushi Road to East Yangzhuang Street and Fushi Road, and the intersection area is the east entrance between East Yangzhuang Street and Fushi Road.

Fig. 1 the schematic of the experiment link

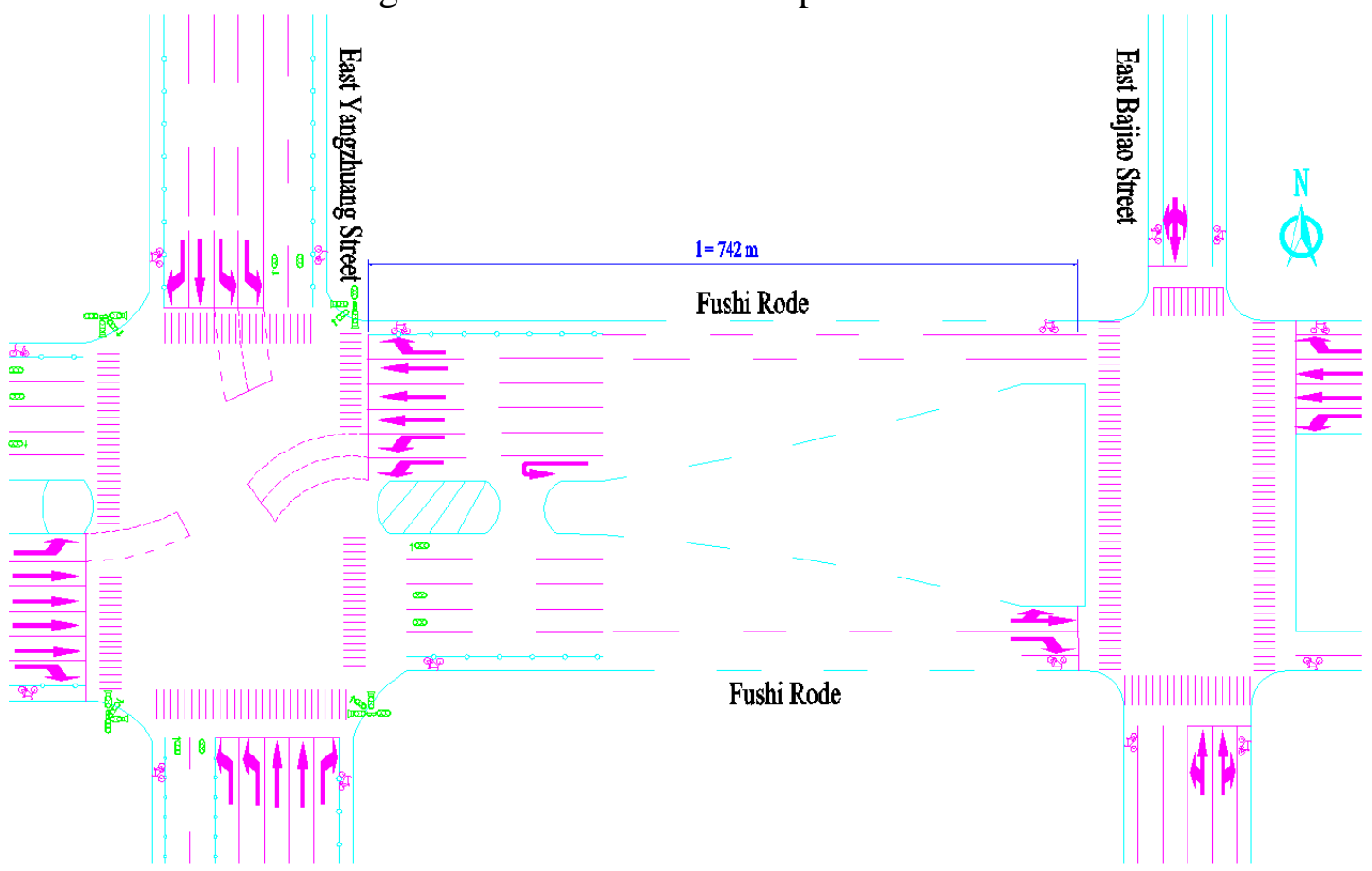

The collected data are:

(1) Green time $t_{g}=35 \mathrm{~s}$, Yellow light time $t_{y}=4 \mathrm{~s}$, Red light time $t_{r}=122 \mathrm{~s}$, Yellow and red light time $t_{a}=126 \mathrm{~s}$.

(2) Loss of time $t_{l}=3 \mathrm{~s}$.

(3) The number of lanes of the import lane $n=3$, the $x$ lane time headway $h_{x}$.

(4) Import Road Capacity $Q=300 p c u$, which allows the largest number of vehicles queuing

(5) The maximum number of vehicles crossing the intersection during the effective green time $s=80 p c u$.

Table 1 is the law of arrival at green time and Table 2 is the law of arrival at yellow, red light time. 
Table 1

\begin{tabular}{cc}
\hline Number of arriving vehicles & Probability of arriving vehicles \\
\hline 0 & 0.0968 \\
1 & 0.0645 \\
2 & 0.0323 \\
3 & 0.1613 \\
4 & 0.1935 \\
5 & 0.0645 \\
6 & 0.0645 \\
7 & 0.0645 \\
8 & 0.0968 \\
9 & 0.0323 \\
10 & 0.0323 \\
11.5 & 0.0323 \\
12 & 0.0323 \\
\hline
\end{tabular}

Table 2

\begin{tabular}{cc}
\hline Number of arriving vehicles & Probability of arriving vehicles \\
\hline 15 & 0.0323 \\
26 & 0.0968 \\
28 & 0.0968 \\
28.5 & 0.0323 \\
29.5 & 0.0323 \\
30 & 0.0645 \\
31 & 0.0323 \\
31.5 & 0.0645 \\
32 & 0.0323 \\
33 & 0.0323 \\
34 & 0.0968 \\
34.5 & 0.0323 \\
35 & 0.0323 \\
36 & 0.0323 \\
37 & 0.0645 \\
39 & 0.0323 \\
39.5 & 0.0323 \\
40 & 0.0323 \\
41 & 0.0645 \\
44 & 0.0323 \\
47 & 0.0323 \\
\hline
\end{tabular}

The forecast result of the travel time estimation is shown in Fig.2 through programming in Matlab. Verify the model by the floating car data and the results show that the relative absolute error of the model is $20 \%$. 
Fig. 2 the forecast result of the travel time estimation

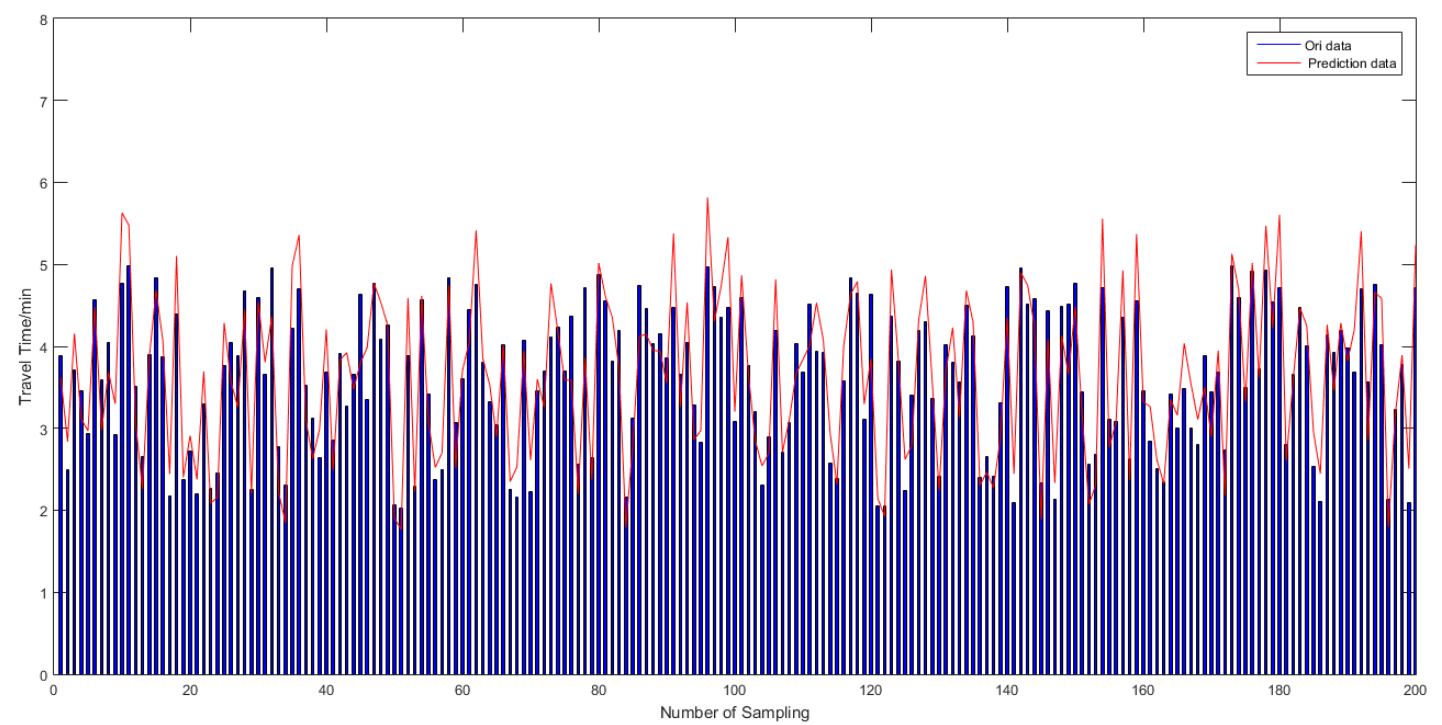

\section{Conclusions}

Based on the Markov chain and traffic wave theory, the travel time estimation model is constructed considering the stochastic vehicle arrival and verified by a practical road in Beijing, which proves that the model has high estimation precision.

The travel time is estimated by simulating the actual traffic flow and appropriate to the intersection with fixed signal cycle, and the travel time prediction is the next research direction because the basis of travel time prediction is the travel time estimation.

\section{Acknowledgements}

This work was financially supported by National Natural Science Foundation of China(70672023), Scientific and technological innovation service capacity program(KM201510009002) and North China University of Technology professional project(XN050).

\section{References}

[1] Turner S M. Advanced Techniques for Travel Time Data Collection. Proceedings of the 6th International Vehicle Navigation and Information Systems Conference and Conjunction with the Pacific Rim TransTech Conference. 1995

[2] H. M. Zhang. Link-Journey-Speed Model for Arterial Traffic. Transportation Research Record: Journal of the Transportation Research Board. 1999

[3] Chu L,Oh S,Recker W.Adaptive Kalman filter based freeway travel time estimation. 84th TRB Annual Meeting . 2005

[4] Frechette L A, Khan A M. Bayesian regression based urban traffic models[J]. Transportation Research Record: Journal of the Transportation Research Board, 1998, 1644(1):157 165

[5] Zhu Z. Wang W. A travel time estimation model for route guidance systems[C]// Proceedings of the 2nd International Conference on Traffic and Transportation Studies. ASCE,2000:547 552

[6] David Anthony faria, A framework to transform real-time GPS derived from transit vehicles to determine speed-flow[D]. Arlington. The university of TEXAS at Arlington. 2003. [6] Innamaa S. 
Short-term prediction of travel time using neural networks on an interurban highway[J]. Transportation, 2005,32(6):649 669

[7] David Anthony faria, A framework to transform real-time GPS derived from transit vehicles to determine speed-flow[D]. Arlington. The university of TEXAS at Arlington. 2003.

[8] Wu Qiao. Travel Time Estimation and Prediction for Urban Road Networks[D]. Zhejiang University.2015

[9] Gerolimins N, Skabardonis A. Identification and analysis of queue spillovers in city street networks[J]. IEEE Transactions on Intelligent Transportation Systems, 2011,12(4):1107 1115

[10] Liu H X, et al. Real-time estimation of arterial travel time under congested conditions[J]. Transportmetrica,2012,8(2):87 104

[11] JIANG Yangsheng, et al. Markov Chain Queuing Model for Signalized Intersections with Stochastis Vehicle Arrivals[J]. Journal of Southwest Jiaotong University, 2010,45(4):621 626

[12] TANG Keshuang, DONG keran, et al. A Comparative Study of Queue Discharge Characteristics at Signalized Intersection[J]. Journal of Tongji University(Natural Science), 2015, 43(11):1689-1695 